\title{
Sensitivity of GPS and GLONASS orbits with respect to resonant geopotential parameters
}

\author{
D. Ineichen, G. Beutler, U. Hugentobler \\ Astronomical Institute, University of Berne, Sidlerstrasse 5, 3012 Bern, Switzerland \\ e-mail: daniel.ineichen@swisstopo.ch; Tel.: +41-31-9632151; Fax: +41-31-9632459
}

Received: 14 October 2002 / Accepted: 9 April 2003

\begin{abstract}
The Center for Orbit Determination in Europe (CODE) has been involved in the processing of combined GPS/GLONASS data during the International GLONASS Experiment (IGEX). The resulting precise orbits were analyzed using the program SORBDT. Introducing one satellite's positions as pseudo-observations, the program is capable of fitting orbital arcs through these positions using an orbit improvement procedure based on the numerical integration of the satellite's orbit and its partial derivative with respect to the orbit parameters. For this study, the program was enhanced to estimate selected parameters of the Earth's gravity field. The orbital periods of the GPS satellites are - in contrast to those of the GLONASS satellites $-2: 1$ commensurable $\left(P_{\mathrm{Sid}}: P_{\mathrm{GPS}}\right)$ with the rotation period of the Earth. Therefore, resonance effects of the satellite motion with terms of the geopotential occur and they influence the estimation of these parameters. A sensitivity study of the GPS and GLONASS orbits with respect to the geopotential coefficients reveals that the correlations between different geopotential coefficients and the correlations of geopotential coefficients with other orbit parameters, in particular with solar radiation pressure parameters, are the crucial issues in this context. The estimation of the resonant geopotential terms is, in the case of GPS, hindered by correlations with the simultaneously estimated radiation pressure parameters. In the GLONASS case, arc lengths of several days allow the decorrelation of the two parameter types. The formal errors of the estimates based on the GLONASS orbits are a factor of 5 to 10 smaller for all resonant terms.
\end{abstract}

Keywords: Global positioning system - GLONASS Orbit determination - Resonant geopotential coefficients

Correspondence to: D. Ineichen

Present address: Bundesamt fuer Landestopografie,

Seftigenstrasse 264, 3084 Wabern, Switzerland

\section{Introduction}

CODE, the Center for Orbit Determination in Europe, is a cooperation between the Astronomical Institute of the University of Berne (AIUB), Berne (Switzerland), the Swiss Federal Office of Topography (swisstopo), Wabern (Switzerland), the Federal Agency for Cartography and Geodesy (BKG), Frankfurt (Germany), and the Institut Géographique National (IGN), Paris (France). Since the commencement of the International GPS Service (IGS) in June 1992, CODE has been one of the eight IGS Analysis Centers, and processes GPS observations of a global network on a daily basis (Rothacher et al. 1999; Hugentobler et al. 2002).

The Bernese GPS Software was enhanced to process GLONASS data (Habrich 1999; Hugentobler et al. 2001) and CODE participated as one of several analysis centers in the International GLONASS Experiment (IGEX) which started in October 1998. Information about the organization, the goals, and the results obtained from the IGEX campaign may be found in, for example, Ineichen et al. (1999), Slater et al. (1999), and Willis et al. (1999). CODE was involved in the processing of combined GPS and GLONASS data and the computation of precise GLONASS orbits.

Unfortunately, the GLONASS system is far from complete and the number of active satellites decreased from 15 in June 1999, to 7 in May 2002. Due to this development, CODE decided to suspend the computation of precise GLONASS orbits and postponed the proposed merging of the IGS and the IGEX routine processing procedures (Ineichen et al. 2001). Instead, emphasis was put on the analysis of the GLONASS orbits generated during the official IGEX campaign. In addition, there are ongoing studies and developments at CODE in the field of satellite orbit modeling for low Earth orbiters (LEOs) (see Bock et al. 2003). The program SORBDT may be used for both LEO processing 
and estimation of parameters of the Earth's gravity field using GPS and GLONASS ephemerides.

This paper is a continuation of previous studies performed at CODE in the field of orbit modeling and geopotential parameter estimation. Beutler et al. (1994) performed estimates of resonant low-order terms of the Earth's gravity field using GPS data. Hugentobler (1998) and Hugentobler et al. (1999) estimated the terms $S_{22}$ and $C_{22}$ using astrometric observations of geostationary satellites, as well as comparing the drift rates observed for the semi-major axis of GPS satellites with the values expected from theory.

Here, the sensitivity of the GPS and the GLONASS orbits with respect to a few selected parameters of the Earth's gravity field is analyzed. It was expected that, in particular, the GPS orbits would be well suited for deriving estimates for resonant geopotential parameters. The inclusion of GLONASS ephemerides permits the study of the impact of the orbital characteristics of GPS and GLONASS on the determination of resonance parameters. Differences in behavior were expected due to the differences in the orbital periods (the GLONASS satellites are not in deep 2:1 resonance with the Earth's rotation), differences in the inclination angles of the orbital planes (55.0 degrees for GPS, compared to 64.8 degrees for GLONASS), and differences in the behavior of GPS and GLONASS satellites regarding the Y-bias (no significant Y-bias is observed for the GLONASS satellites; see Ineichen et al. 2001).

The influence of the inclination angle and revolution period on the ground tracks of a GPS and a GLONASS satellite is illustrated in Fig. 1. Whereas the GPS satellites have revolution periods of half a sidereal day, the GLONASS satellites have revolution periods of 8/17 of a sidereal day. This implies that the GPS satellites are at the same position with respect to an Earth-fixed reference system after 1 sidereal day (two orbital revolutions). The GLONASS satellites, however, perform $2 \frac{1}{8}$ revolutions in 1 sidereal day and do not appear at the same position after 1 sidereal day. They repeat their ground tracks only after 8 days. Therefore, GLONASS satellite orbits do not exhibit a deep resonance behavior.

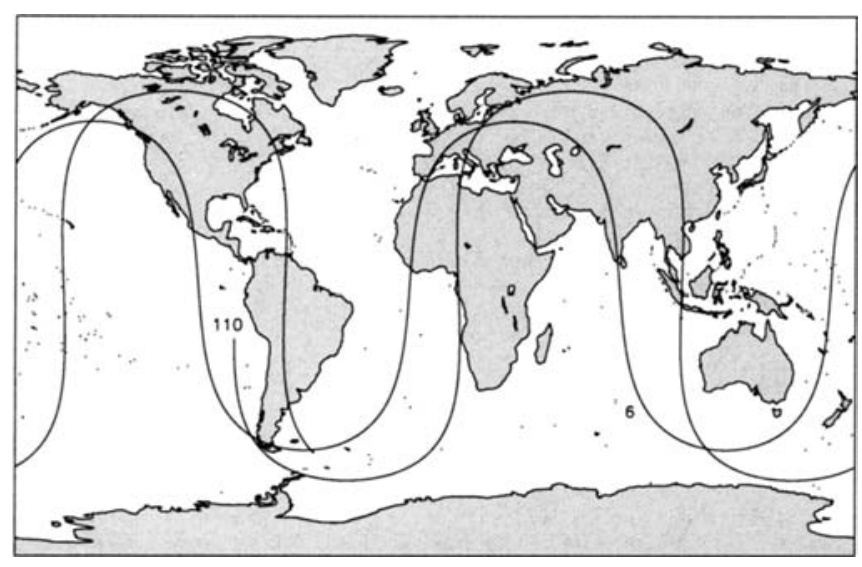

Fig. 1. Ground tracks of a GPS satellite (6) and a GLONASS satellite (110) for the time interval of 1 sidereal day

\section{Resonance}

Lagrange's planetary equation for perturbations in the semi-major axis due to the geopotential is (Kaula 1966)

$\frac{\mathrm{d} a}{\mathrm{~d} t}=-2 n a \sum_{l m p q}\left(\frac{a_{e}}{a}\right)^{l}(l-2 p+q) F_{l m p} G_{l m q} J_{l m} \sin \Psi_{l m p q}$

with

$\Psi_{l m p q}=(l-2 p) \omega+(l-2 p+q) M+m\left(\Omega-\Theta-\lambda_{l m}\right)$

where $a, n, \Omega, \omega$, and $M$ denote the semi-major axis, mean motion, right ascension of the ascending node, argument of perigee, and mean anomaly of the satellite. $\Theta$ is the sidereal time, $a_{e}$ the equatorial radius of the Earth, $J_{l m}$ the geopotential coefficient of degree $l$ and order $m$, and $\lambda_{l m}$ the corresponding reference longitude. $F_{l m p}$ and $G_{l m q}$ represent the so-called inclination function and eccentricity function, respectively. The index $p$ runs from zero to degree $l$ and the index $q$ from minus to plus infinity.

Resonant perturbation occurs if the argument $\Psi_{l m p q}$ of the trigonometric function is time independent. This condition may be met for certain combinations of the indices $l, m, p$, and $q$ for satellite revolution periods commensurable with the Earth's rotation (Klokocnik 1983). For GPS with a mean motion which is twice the sidereal rate, $n=\dot{M} \simeq 2 \dot{\Theta}$, the condition for resonance is:

$(l-2 p+q) 2 \simeq m$

The resulting perturbations are periodic with periods of several years, and are the primary reason for most of the maneuvers of the GPS satellites.

Table 1 summarizes the most important resonant parameters causing significant drift rates for the semimajor axis of the GPS satellite orbits. The most important resonant term is $J_{32}$ (maximum drift rate in $a$ of $6.0 \mathrm{~m} /$ day $)$, followed by $J_{44}(1.5 \mathrm{~m} /$ day $)$, and $J_{22}(0.6$ and $0.4 \mathrm{~m} /$ day). In the case of GLONASS orbits, these parameters do not cause a constant drift in the semimajor axis $a$, but perturbations with periods of between 2 and 8 days. The periods and amplitudes are included in Table 1 .

\section{Approach}

For the assessment of the sensitivity of the GPS and the GLONASS orbits with respect to the geopotential coefficients, precise orbit positions (Cartesian coordinates each 15 minutes) were introduced as pseudoobservations in the program SORBDT. The input files were generated on the one hand within CODE's IGS routine processing procedure for the GPS satellites, and on the other hand by CODE's IGEX routine processing for the GLONASS satellites.

For the GPS orbits, five radiation pressure parameters of the Extended CODE Orbit Model were estimated and stochastic parameters were set up every 12 hours 
Table 1. Geopotential parameters causing the largest resonance effects for GPS orbits with eccentricity $e=0.006$ (maximum drift rates in the semi-major axis $a$ ) and the corresponding periods and amplitudes of the distortions of the semi-major axis $a$ for GLONASS orbits

\begin{tabular}{llrrlll}
\hline Degree $l$ & Order $m$ & $p$ & $q$ & $\begin{array}{l}\text { Max drift in } a \\
\text { (GPS) (m/day) }\end{array}$ & $\begin{array}{l}\text { Period in } a \\
\text { (GLONASS) (days) }\end{array}$ & $\begin{array}{l}\text { Amplitude in } a \\
\text { (GLONASS) (m) }\end{array}$ \\
\hline 3 & 2 & 1 & 0 & 6.046 & 7.97 & 3.269 \\
4 & 4 & 1 & 0 & 1.484 & 3.99 & 1.130 \\
2 & 2 & 1 & 1 & 0.635 & 7.97 & 0.261 \\
2 & 2 & 0 & -1 & 0.390 & 7.97 & 0.107 \\
5 & 2 & 2 & 0 & 0.227 & 7.97 & 0.313 \\
6 & 4 & 2 & 0 & 0.022 & 3.99 & 0.057 \\
7 & 6 & 2 & 0 & 0.017 & 2.66 & 0.000 \\
4 & 2 & 2 & 1 & 0.014 & 7.97 & 0.001 \\
\hline
\end{tabular}

(Beutler et al. 1994; Springer et al. 1999). The Earth's gravity field was represented by the JGM-3 model (Tapley et al. 1996) and assumed known, and the solid Earth tides and the ocean tides were taken into account. The gravitational attractions of the Sun and Moon were included. Tropospheric influences were accounted for by estimating 2-hourly tropospheric parameters, and the effects of the ionosphere were eliminated by using the ionosphere-free $L_{3}$ linear combination. Satellite clock errors and station clock errors were eliminated by forming double-differenced observations.

For the generation of the GLONASS orbits, CODE's precise GPS orbits and Earth orientation parameters were kept fixed and the solutions were aligned to the ITRF 97 (Boucher et al. 1999). In contrast to the procedure for the GPS orbits, in this case all nine radiation pressure parameters of the Extended CODE Orbit Model were set up. The final solution for 1 day corresponds to the middle day of a 5-day arc. Long arc fits, comparisons with the precise orbits of other analysis centers, and validation of the orbits with SLR measurements indicated that the orbits are on a $10-20 \mathrm{~cm}$ accuracy level. Therefore, they are about four times more inaccurate than the GPS orbits, due mainly to the lower number of sites tracking GLONASS data. More details concerning the generation of the precise GLONASS orbits may be found in Ineichen et al. (1999).

The results for the geopotential parameters and their variance/covariance information presented here were determined as follows. Through the above mentioned orbits, 1-day or several-day arcs were generated using the positions of the satellites as pseudo-observations. The following parameters were solved for in the orbit determination procedure.

1. Six osculating elements (semi-major axis $a$, numerical eccentricity $e$, inclination of the orbital plane $i$, right ascension of the ascending node $\Omega$, argument of perigee $\omega$, and argument of latitude $u_{0}$ referring to the initial epoch $t_{0}$ ).

2. All nine radiation pressure parameters of the Extended CODE Orbit Model: three constant terms in the direction from the satellite to the Sun $\left(D_{0}\right)$, the spacecraft's solar panel axis $\left(Y_{0}\right)$, and the third direction completing a Cartesian right-handed system $\left(X_{0}\right)$, plus six periodic terms, cosine and sine terms in each of these directions.

3. A subset of the geopotential coefficients.
The orbit determination is carried out satellite by satellite in the program SORBDT, and for each satellite the above-mentioned parameters are solved for. After the loop over all satellites, three different values for the estimated potential parameters are derived: one for all GPS satellites, one for all GLONASS satellites, and one using all GPS and GLONASS satellites. In addition, the program SORBDT is able to save these combined values together with the variance/covariance information in reduced normal equation files, where all other parameter types are pre-eliminated. An additional program was developed which allows for the stacking of several solutions based on the principles of manipulating and stacking normal equation systems (Brockmann 1996).

It might be argued that the geopotential parameters estimated by this approach would be the same as the values of the JGM-3 a priori gravity model used for the generation of the input orbits. This is actually true for 1day orbits. In the case of longer orbital arcs, however, it is possible to obtain estimates of the geopotential parameters which are in essence independent of the a priori gravity model (see Sect. 5.1).

\section{Variance/covariance analysis}

In this section we analyze the variance/covariance information of the geopotential parameters estimated according to the method described in Sect. 3. In particular, we study the behavior of a resonant term in contrast to a non-resonant term, on the one hand estimated using GPS orbits and on the other hand estimated using GLONASS orbits. We also show the resulting correlations between the radiation pressure parameters and the estimated geopotential parameters in the case of a GPS satellite and in the case of a GLONASS satellite.

\subsection{Formal accuracy of $S_{32}$ and $S_{33}$}

Let us start with the formal accuracy of the estimated geopotential parameters. We compare the results from a 1-day arc (day 121, 2000) with the results from a 7-day arc (days 121-127, 2000). Figure 2 shows the results for the most important resonant sine term $\left(S_{32}\right)$ and a nonresonant sine term $\left(S_{33}\right)$. The formal errors are based on the assumption that the input orbits have a mean error 

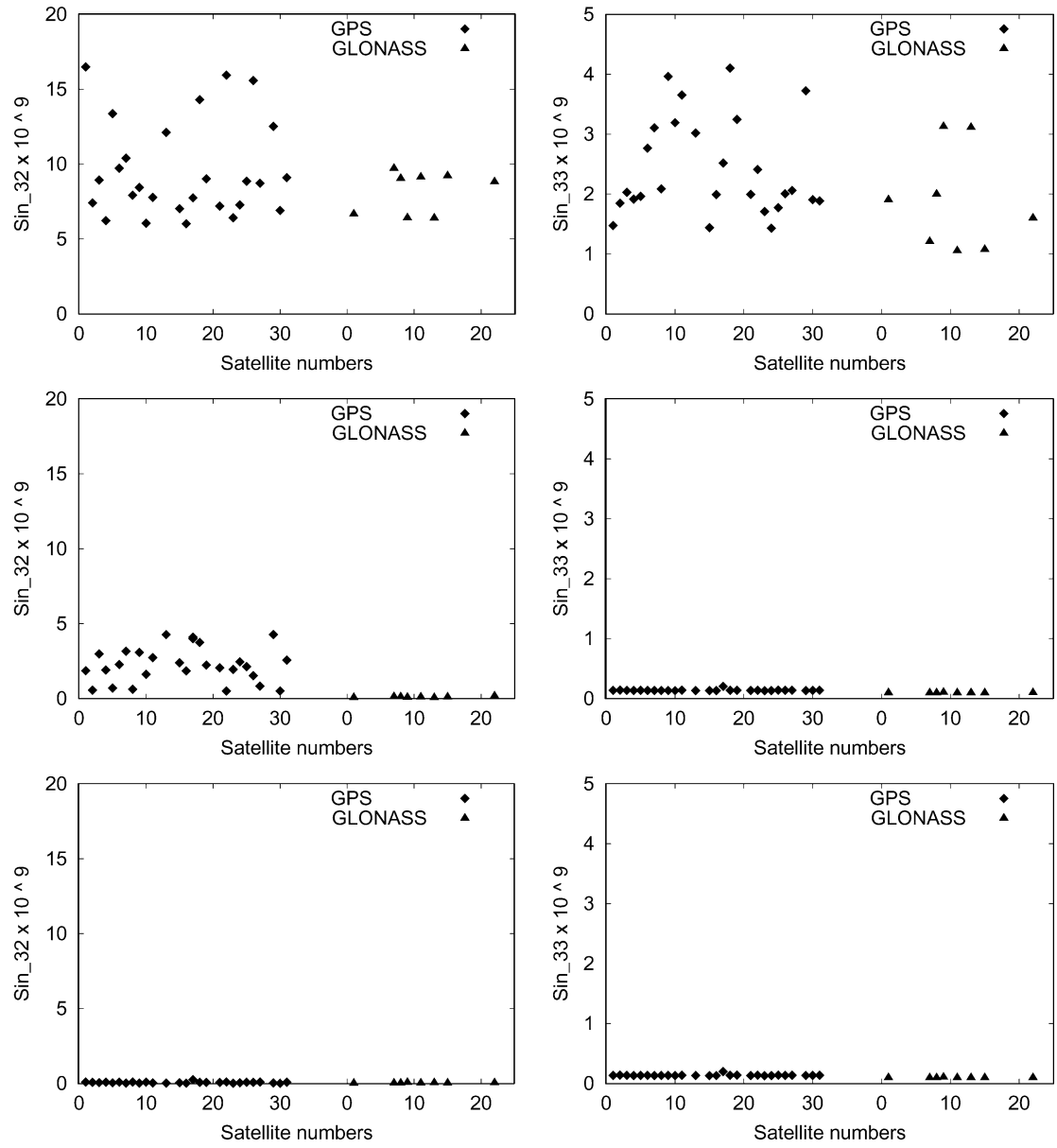

Fig. 2. A priori RMS of the estimated parameters. Sine term of degree 3 and order 2 (left, resonant term) and sine term of degree 3 and order 3 (right, non-resonant term). One-day solution estimating radiation pressure parameters (top), 7-day solution estimating radiation pressure parameters (middle), and 7-day solution without estimating any radiation pressure parameters (bottom)

of $3 \mathrm{~cm}$ (which is appropriate for the GPS orbits, but somewhat optimistic for the GLONASS orbits).

For the 1-day arcs, the formal errors of the parameters derived by GPS orbits are comparable to those derived by GLONASS: $5 \cdot 10^{-9}$ to $16 \cdot 10^{-9}$ for the $S_{32}$ term and $1 \cdot 10^{-9}$ to $4 \cdot 10^{-9}$ for the $S_{33}$ term.

When these values are compared with the results stemming from a 7-day arc, we note that in the case of the resonant term $\left(S_{32}\right)$, the formal errors for the parameters derived using GPS satellites are completely different to those derived using GLONASS satellites. The GPS-derived errors are of the order of $5 \cdot 10^{-9}$, whereas the GLONASS-derived estimates have errors which are all below $0.2 \cdot 10^{-9}$. This is a surprising result, as we would expect the GPS satellites to be particularly sensitive to the resonant parameters. In the case of the non-resonant sine term $\left(S_{33}\right)$, the formal errors are below $0.2 \cdot 10^{-9}$ for all satellites and are about the same for the GPS-derived and the GLONASS-derived values.

Figure 2 shows also the formal errors of the geopotential parameters derived by a 7-day arc if no radiation pressure parameters are estimated. In fact, the formal errors of the resonant parameter $\left(S_{32}\right)$ decrease to $0.1 \cdot 10^{-9}$ for all GPS satellites and are now comparable to the values derived by GLONASS satellites. This is a clear indication that the correlation of the gravity field parameters with the radiation pressure parameters dis- turbs the estimation of the resonant parameter $J_{32}$, in particular when using GPS orbits.

The so-called Y-bias, i.e. the acceleration along the solar panels, is responsible for a perturbation containing a component with an annual period. The Y-bias thus generates a drift in the semi-major axis which is almost constant over weeks and has a similar effect to a resonant geopotential parameter. The two effects are therefore hardly separable when using only GPS orbits. Hugentobler (1998) has shown that the Y-bias induces a drift rate on the semi-major axis of up to almost $20 \%$ of the effect stemming from the resonant geopotential parameters. The GLONASS satellites are, in contrast to the GPS satellites, not in deep 2:1 resonance with the Earth's rotation. As a consequence, the resonant parameters induce perturbations with periods of only 2 to 8 days. Therefore, the influences of the radiation pressure parameters and the resonant geopotential parameters can be separated much better when analyzing orbital arcs of several days. GLONASS orbits are therefore much better suited for the estimation of resonant geopotential parameters than are GPS orbits.

\subsection{Correlation information}

The correlation of the estimated geopotential parameters with the radiation pressure parameters is one of the 
crucial issues. Therefore, correlation matrices provide valuable information on the correlations between the estimated parameters. In the parameter estimation process six orbital elements $\left(a, e, i, \Omega, \omega\right.$, and $\left.u_{0}\right)$, nine radiation pressure parameters $\left(D_{0}, Y_{0}, X_{0}, D_{C}, D_{S}, Y_{C}, Y_{S}\right.$, $X_{C}$, and $X_{S}$ ), and all geopotential parameters simultaneously up to degree and order $6\left(C_{20}, S_{21}, C_{21}, S_{22}\right.$, $C_{22}, \ldots$ ) are set up.

The correlation coefficient $\rho$ of two estimated parameters $x$ and $y$ is defined as

$\rho=\frac{\operatorname{cov}(x, y)}{\sigma_{x} \cdot \sigma_{y}}$

where $\operatorname{cov}(x, y)$ is the covariance of parameters $x$ and $y$, and $\sigma_{x}$ and $\sigma_{y}$ are the standard deviations of parameters $x$ and $y$.

Figure 3 illustrates the correlation coefficients for GPS satellite 4 (left) and GLONASS satellite 1 (right), based on a 1-day arc (day 121, 2000). The upper left triangle contains the relevant information, the absolute values of the correlation coefficients. Dark means low correlation (correlation coefficients close to zero), bright means high correlation (absolute value of the correlation coefficients approaching 1). The diagonal elements must be white (because the correlation of a parameter with itself is 1). The lower right black triangles should be ignored (symmetric to the upper triangular part). The correlation diagram for the GPS satellite shows a clear pattern of high correlations between some of the parameters. The following aspects should be noted.

1. There is a high degree of correlation of the geopotential terms $J_{20}, J_{32}, J_{40}, J_{44}, J_{52}, J_{60}$, and $J_{64}$ with the radiation pressure parameters $Y_{0}, X_{0}, D_{C}$, and $D_{S}$. The terms are either zonal terms with even degree $\left(J_{20}, J_{40}\right.$, and $\left.J_{60}\right)$ or resonant terms $\left(J_{32}, J_{44}, J_{52}\right.$, and $\left.J_{64}\right)$.

2. There is a high degree of correlation between various geopotential parameters. As expected, all geopoten- tial parameters highly correlated with the radiation pressure parameters also show correlations between themselves.

3. Correlations are also clearly visible for the so-called lumped coefficients (Gooding 1971). As an example, the correlation of $J_{22}$ with $J_{42}$ and $J_{62}$, clearly visible in Fig. 3 for GPS satellite 4, may be studied.

It is interesting to note that the corresponding correlation matrix of a GLONASS satellite does not reveal similar patterns.

\section{Estimated parameters}

\subsection{Proof of concept}

In this section we prove that it is possible to estimate geopotential parameters with the method proposed in this paper. We re-computed the input orbits of the GPS satellites for a time span of 9 days (days 116-124, 2002), introducing biased a priori information for the geopotential. The test was performed for resonant terms $\left(S_{32}\right.$ and $\left.C_{32}\right)$ and non-resonant terms $\left(S_{33}\right.$ and $\left.C_{33}\right)$. The following biases were applied to the JGM-3 a priori gravity model.

1. $S_{32}$ changed from $-619 \cdot 10^{-9}$ to $-630 \cdot 10^{-9}$.

2. $C_{32}$ changed from $905 \cdot 10^{-9}$ to $890 \cdot 10^{-9}$.

3. $S_{33}$ changed from $1414 \cdot 10^{-9}$ to $1400 \cdot 10^{-9}$.

4. $C_{33}$ changed from $721 \cdot 10^{-9}$ to $700 \cdot 10^{-9}$.

The 1-day orbits were generated using a very similar processing scheme as used for the generation of CODE's IGS rapid products (see Springer 2000; Schaer 1999).

In a second step, these GPS orbits were used as input orbits for fitting 1- to 9-day arcs and estimating the values for the geopotential parameters which were biased in the input orbits. The goal was to check whether the estimated parameters stay close to the biased a priori
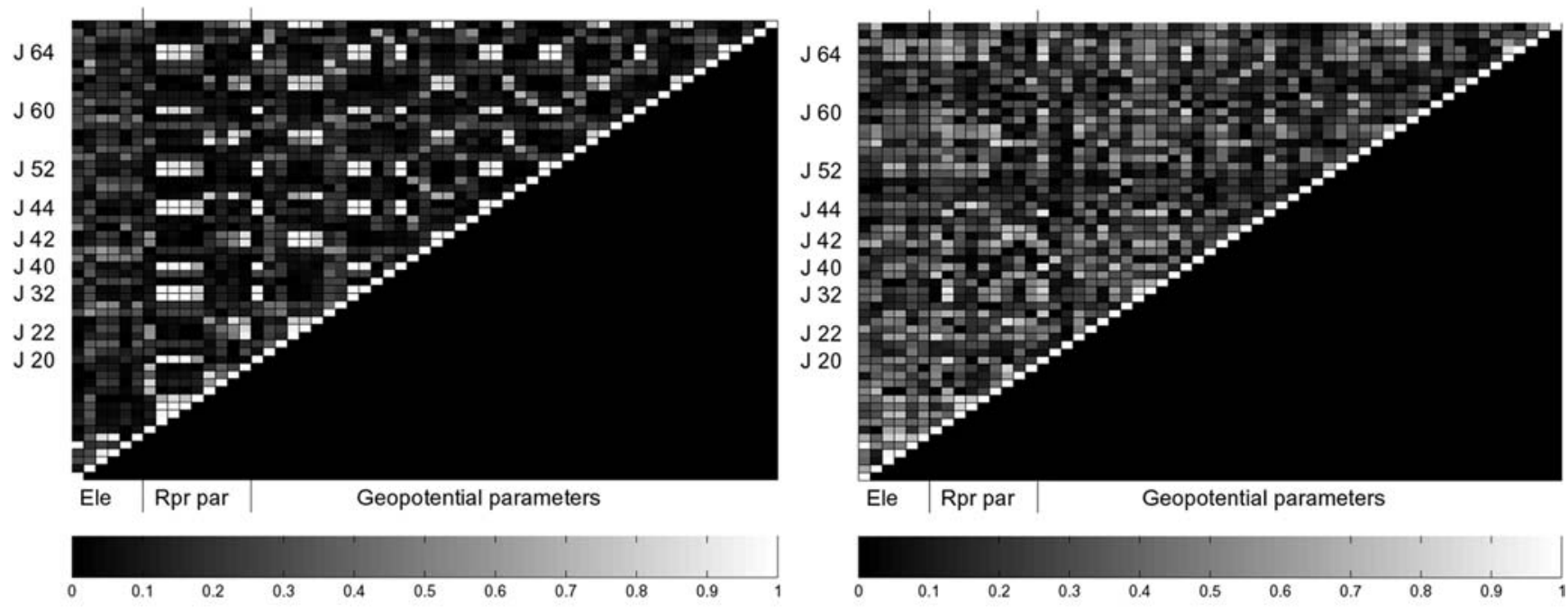

Fig. 3. Correlation between parameters estimated by GPS satellite 4 (left) and by GLONASS satellite 1 (right), day of year 121, 2000. The set-up parameters are six orbital elements (Ele), nine radiation pressure parameters (Rpr par), and 45 geopotential parameters up to degree and order 6 

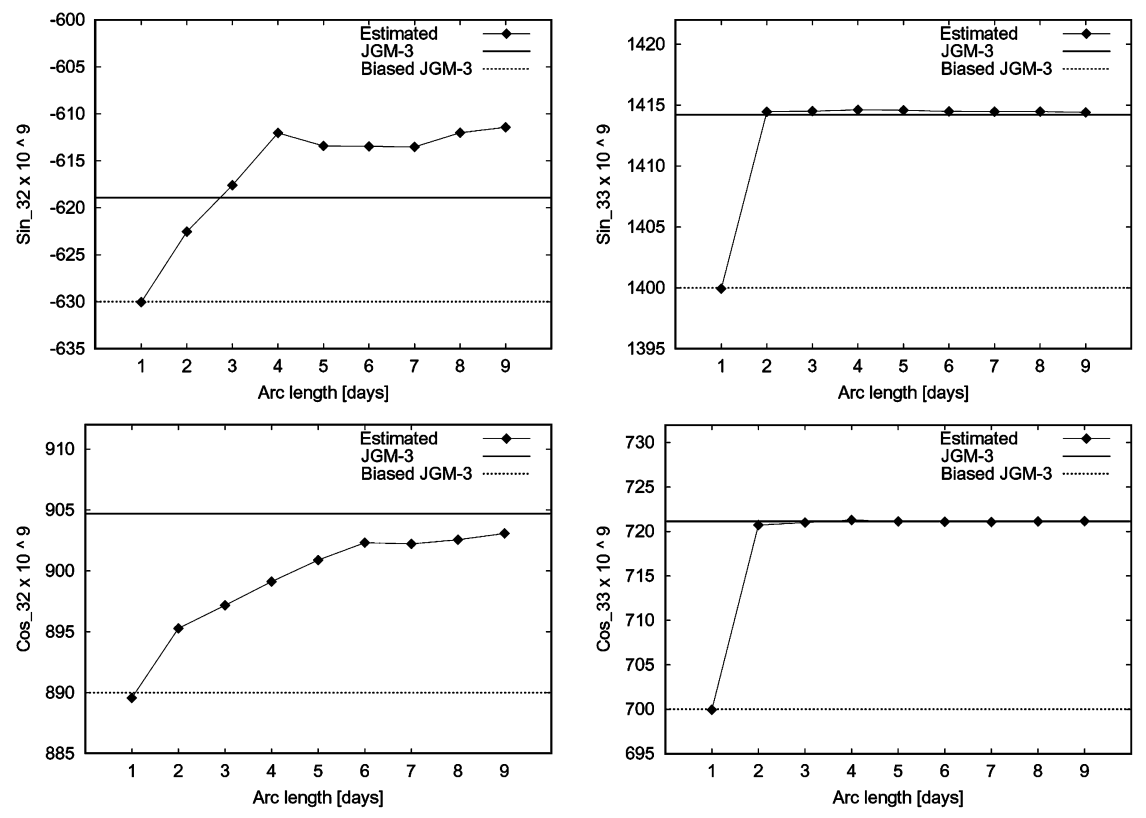

Fig. 4. Estimates of geopotential parameters based on 1- to 9-day fits through GPS input orbits generated with biased a priori gravity model. Resonant terms of degree 3 and order 2 (left) and non-resonant terms of degree and order 3 (right). Sine terms (top) and cosine terms (bottom) geopotential or whether they adopt reasonable values, i.e. values close to the JGM-3 values.

Five GPS satellites were excluded from the combination, namely the satellites $2,14,17$, and 21 , because at least 1 day of the 9-day time interval is missing in $\mathrm{CO}$ DE's orbit products. Satellite 29 was left out because its orbit fit on the long-arc level yielded an RMS five times higher than that of the other satellites. Figure 4 shows the combined estimates based on GPS orbits for the resonant terms $S_{32}$ and $C_{32}$ (left) and the non-resonant terms $S_{33}$ and $C_{33}$ (right). The estimates are shown as a function of the arc length (1- to 9-day arcs). In addition, the horizontal lines on the plots represent the biased a priori information (lower line) and the correct JGM-3 value (upper line), respectively.

When inspecting the results based on the 1-day arcs, we recognize that - as anticipated - the estimates of all four parameters are very close to the biased a priori information $\left(-630.0 \cdot 10^{-9}\right.$ for $S_{32}, 889.6 \cdot 10^{-9}$ for $C_{32}$, $1399.9 \cdot 10^{-9}$ for $S_{33}$, and $699.9 \cdot 10^{-9}$ for $C_{33}$ ).

If we extend the length of the orbital arcs, the estimates show a different behavior for the resonant terms and the non-resonant terms. In the case of the resonant terms, the estimates are moving towards the JGM-3 value. The differences at the 9-day level are, however, quite large $\left(7.5 \cdot 10^{-9}\right.$ for $S_{32}$ and $-1.6 \cdot 10^{-9}$ for $\left.C_{32}\right)$. If we started the same procedure from a different day, the resulting graphs might look quite different. The reason is that we used only GPS orbits, which do not allow the appropriate de-correlation of the estimates of the $S_{32}$ and $C_{32}$ terms and the radiation pressure parameters. The additional use of GLONASS satellites will considerably improve the results for these parameters (see Sect. 4).

For the non-resonant terms $C_{33}$ and $S_{33}$ we observe a different behavior: as soon as we extend the orbital arc length to 2 days a very clear movement towards the JGM-3 value is observed. The estimated values jump from $1399.93 \cdot 10^{-9}$ to $1414.45 \cdot 10^{-9}$ for the $S_{33}$ term and from $699.94 \cdot 10^{-9}$ to $720.72 \cdot 10^{-9}$ for the $C_{33}$ term.
The estimated values stay close to the JGM-3 value for all longer arc lengths. For the 9-day arcs, the differences with respect to the JGM-3 values amount to $0.001 \cdot 10^{-9}$ $\left(S_{33}\right)$ and $0.198 \cdot 10^{-9}\left(C_{33}\right)$ with corresponding formal errors of $0.050 \cdot 10^{-9}$. These small differences-even if we drastically bias the a priori information by about $15 \cdot 10^{-9}$ for $S_{33}$ and $21 \cdot 10^{-9}$ for $C_{33}$ - are a clear indicator that it is possible to estimate geopotential parameters by the proposed method.

\subsection{Results}

Using the unbiased JGM-3 model as a priori information, we compare the estimates of a non-resonant geopotential term with the estimates of a resonant term. We select the sine term of degree and order 3 as an example of a non-resonant term and the sine term of degree and order 4 as an example of a resonant term. Figure 5 shows the estimates of $S_{33}$ (left) and $S_{44}$ (right). The results are based on 1-day arcs of day 121 of the year 2000 (top) and on 7-day arcs of days 121-127 of the year 2000 (bottom). The estimated values are given for each individual satellite together with the associated formal error (GPS satellites on the left, GLONASS satellites on the right).

In the case of the non-resonant term $\left(S_{33}\right)$, the scatter of the estimates is a bit smaller for the GLONASS satellites than for the GPS satellites, but all in all comparable for both the 1-day and the 7-day arcs. The noise around the mean value and the formal accuracy is improved when extending the arc length to 7 days. We observe a totally different behavior for the resonant term: the scatter of the estimates based on the GPS orbits is not significantly improved by extending the arc length from 1 to 7 days. The scatter of the estimates based on the GLONASS satellites, however, is reduced by a factor of three when switching to 7-day arcs and is nine times lower compared to GPS. This is even more remarkable considering the 

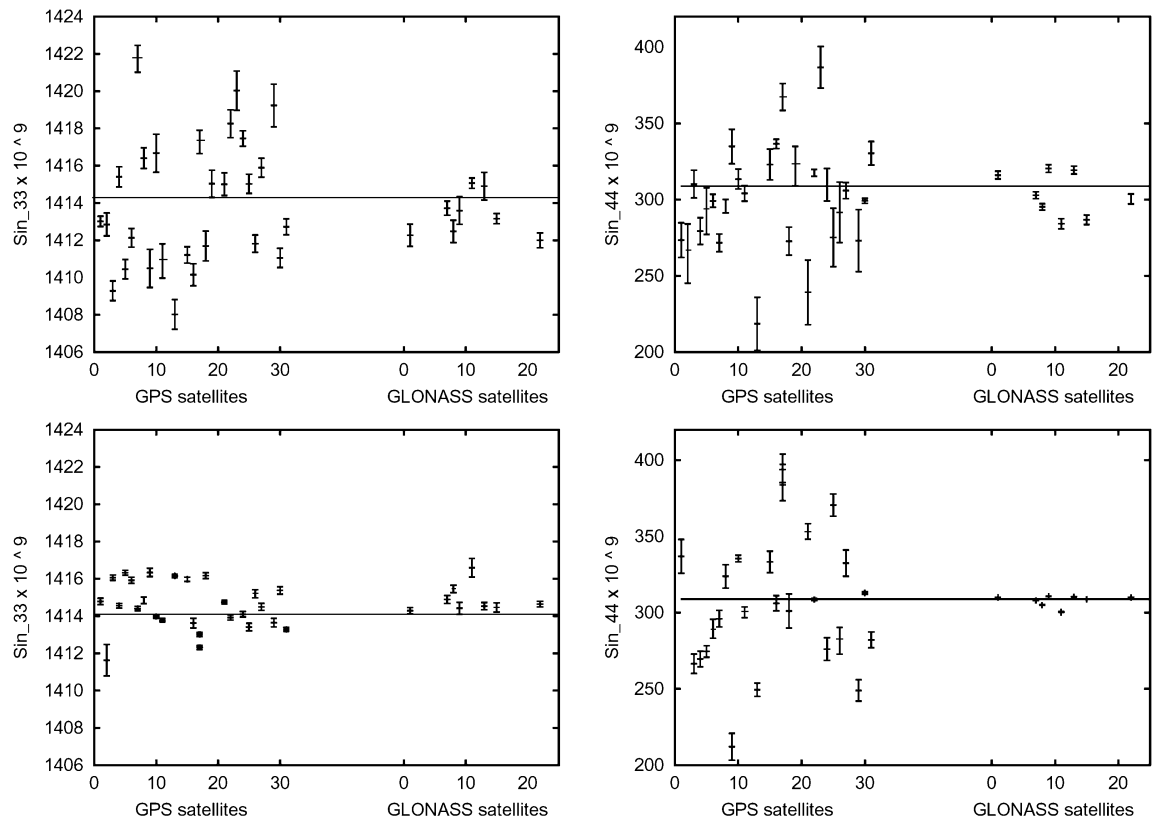

Fig. 5. Estimates geopotential parameters with formal errors for sine term of degree 3 and order 3 (left, non resonant term) compared to the estimates and formal errors for sine term of degree 4 and order 4 (right, resonant term). One-day solution (top) compared to 7-day solution (bottom). Horizontal line: JGM-3 value

Table 2. Estimates and formal errors of the geopotential parameters up do degree and order 4 based on 7-day arcs covering days 121-127, 2000. Given are the JGM-3 value with its formal error and the differences between estimates and the JGM-3 model together with the formal errors for the cases of using all satellites (ALL), GPS satellites only (GPS), and GLONASS satellites only (GLO)

\begin{tabular}{|c|c|c|c|c|c|c|c|c|}
\hline Parameter & JGM-3 & RMS JGM-3 & ALL-JGM-3 & RMS ALL & GPS-JGM-3 & RMS GPS & GLO-JGM-3 & RMS GLO \\
\hline $\cos _{20}$ & -484169.548 & 0.047 & 0.826 & 0.047 & 0.286 & 0.057 & 1.433 & 0.092 \\
\hline $\sin _{21}$ & 1.195 & - & 0.014 & 0.020 & 0.077 & 0.020 & -0.263 & 0.064 \\
\hline $\cos _{21}$ & -0.187 & - & -0.247 & 0.020 & -0.261 & 0.020 & -0.187 & 0.064 \\
\hline $\sin _{22}$ & -1400.266 & 0.037 & 0.200 & 0.021 & 0.198 & 0.142 & 0.192 & 0.028 \\
\hline $\cos _{22}$ & 2439.263 & 0.037 & -0.476 & 0.022 & 2.194 & 0.135 & -0.533 & 0.029 \\
\hline $\cos _{30}$ & 957.176 & 0.036 & 25.766 & 0.662 & 29.937 & 0.694 & 16.817 & 1.591 \\
\hline $\sin _{31}$ & 248.131 & 0.115 & 0.887 & 0.295 & 0.458 & 0.301 & 2.036 & 0.763 \\
\hline $\cos _{31}$ & 2030.137 & 0.115 & -2.618 & 0.288 & -2.531 & 0.291 & -2.863 & 0.764 \\
\hline $\sin _{32}$ & -618.923 & 0.094 & -0.865 & 0.046 & 1.710 & 0.280 & -0.917 & 0.061 \\
\hline $\cos _{32}$ & 904.706 & 0.094 & -2.290 & 0.044 & 0.833 & 0.285 & -2.348 & 0.058 \\
\hline $\sin _{33}$ & 1414.204 & 0.057 & 0.321 & 0.034 & 0.250 & 0.038 & 0.441 & 0.077 \\
\hline $\cos _{33}$ & 721.145 & 0.058 & -0.024 & 0.034 & -0.008 & 0.037 & -0.051 & 0.076 \\
\hline $\cos _{40}$ & 539.777 & 0.134 & 16.367 & 0.746 & 6.251 & 1.966 & 17.615 & 1.058 \\
\hline $\sin _{41}$ & -473.772 & 0.087 & -1.298 & 0.444 & 1.585 & 0.595 & -3.408 & 0.785 \\
\hline $\cos _{41}$ & -536.244 & 0.087 & -3.500 & 0.441 & -4.992 & 0.588 & -2.400 & 0.782 \\
\hline $\sin _{42}$ & 662.571 & 0.156 & -1.943 & 0.315 & 21.432 & 3.030 & -2.142 & 0.407 \\
\hline $\cos _{42}$ & 350.670 & 0.156 & 17.177 & 0.308 & 11.338 & 3.478 & 17.169 & 0.397 \\
\hline $\sin _{43}$ & -200.987 & 0.079 & -0.127 & 0.705 & 0.036 & 0.723 & -0.582 & 1.803 \\
\hline $\cos _{43}$ & 990.869 & 0.079 & 1.094 & 0.712 & 1.937 & 0.733 & -1.065 & 1.801 \\
\hline $\sin _{44}$ & 308.848 & 0.072 & 0.129 & 0.036 & 0.624 & 0.498 & 0.130 & 0.048 \\
\hline $\cos _{44}$ & -188.481 & 0.072 & -0.934 & 0.037 & 6.558 & 0.905 & -0.943 & 0.049 \\
\hline
\end{tabular}

lower quality of the GLONASS orbits compared to the GPS orbits. The same reasons as those provided in Sect. 4.1 may explain this finding.

A summary of the results which are obtained when estimating the geopotential parameters up to degree and order 4 using GPS and GLONASS orbits of one week may be found in Table 2. Table 2 contains the JGM-3 a priori model with formal errors, and the difference of the estimated parameters with respect to JGM-3 together with the corresponding formal errors. Estimated parameters and formal errors are shown in the cases of using all satellites, GPS satellites only, and GLONASS satellites only. The results stem from a solution using orbital arcs covering the period days 121-127, 2000. When comparing the results, it is important to keep in mind that only seven GLONASS satellites, in contrast to 24 GPS satellites, were used for the generation of the results. Thus, the formal error of the combined GLONASS solution is intrinsically a factor of two larger compared to that of GPS. The following outcomes of Table 2 should be noted.

1. The estimated differences to the JGM-3 model range from $-1.9 \cdot 10^{-9}$ to $0.9 \cdot 10^{-9}$ for the sine terms and from $-3.5 \cdot 10^{-9}$ to $25.8 \cdot 10^{-9}$ for the cosine term. The biggest differences are obtained for the terms $C_{30}$ $\left(25.8 \cdot 10^{-9}\right), C_{40}\left(16.4 \cdot 10^{-9}\right)$, and $C_{42}\left(17.2 \cdot 10^{-9}\right)$. 
These parameters show an increased formal error with respect to the other parameters as well.

2. The RMS of the estimated parameters ranges from $0.02 \cdot 10^{-9}$ for $J_{21}$ and $J_{22}$ to $0.75 \cdot 10^{-9}$ for $C_{30}$ when using all satellites. The values might be too optimistic for some of the parameters. The differences of the estimated parameters to the JGM-3 model and the repeatabilities from one week to another support this statement. The formal errors are, however, well suited for comparing the relative sensitivity of the GPS and GLONASS orbits to individual geopotential parameters.

3. If we compare the RMS of the combined GPS results with that of the combined GLONASS results, we note that for all resonant terms $\left(J_{22}, J_{32}, J_{42}\right.$, and $\left.J_{44}\right)$ the RMS is a factor of 5 to 10 smaller for the GLONASS satellites. This is remarkable when taking into account the significantly smaller number of active GLONASS satellites compared to those of GPS. Again, we find a better sensitivity of GLONASS orbits when estimating resonant geopotential terms. For the non-resonant terms the RMS of the estimates derived by GLONASS is two to three times higher, which corresponds to the ratio between the numbers of GPS and GLONASS satellites.

The above results are not the 'final results', as they are based on 1 week of data. A significant improvement would be expected when a long time series of weekly solutions was computed and stacked. The availability of such a time series of weekly solutions would furthermore allow us to analyze the behavior of the estimates over longer time periods and to study, for example, the impact of a changing elevation of the Sun over the orbital planes.

\section{Conclusions}

The results presented here indicate the feasibility of determining resonant geopotential coefficients using precise GPS and GLONASS satellite orbits. This feasibility is demonstrated by changing the a priori geopotential information used for the generation of the input orbits, and then estimating the corresponding potential coefficients using these biased orbits.

The estimation of the resonant geopotential terms is, in the case of GPS, hindered by correlations with the simultaneously estimated radiation pressure parameters. This behavior was not initially expected. It was, on the contrary, assumed that the resonant parameters could be determined with a high accuracy using precise GPS orbits thanks to their commensurability with the Earth's rotation. GLONASS satellites, however, show a better RMS for these parameters. The effects of radiation pressure and resonance are de-correlated for arc lengths of several days. According to the formal errors, which are between 5 and 10 times smaller for the GLONASSbased estimates of the resonant parameters, the satelliteto-satellite repeatability is about 10 times better in the GLONASS case (shown for the $J_{44}$ term).
The results improve with increased arc lengths, as long as the overall orbit model is adequate. For arc lengths of up to 9 days, orbit modeling did not pose serious problems. For most satellites, the orbit fits have RMS values below $5 \mathrm{~cm}$ for the GPS satellites and below $10 \mathrm{~cm}$ for the GLONASS satellites.

The method described in this paper is of course not the optimal method for estimating geopotential parameters. However, it allows an investigation of the sensitivity of the GPS and GLONASS orbits with respect to the low-order potential terms. A preferable approach would be to directly set up these parameters in the general parameter estimation procedure based on the original GPS and GLONASS observables. Constraining the parameters to the JGM-3 a priori model for the official IGS products, but saving the parameters at the normal equation level, would make it possible to estimate and combine the values in a subsequent step by removing the constraints. Furthermore, the analysis of the resulting time series with regard to a changing satellite constellation and other seasonal effects would be very interesting.

Acknowledgments. The authors would like to thank all the organizations involved in the IGS and the IGEX campaign, in particular those operating an IGS or IGEX observation site and providing the indispensable data for precise orbit determination.

Note added in proof. In June 2003 (GPS week 1222), CODE resumed the routinely computation of precise GLONASS orbits for the 9 active GLONASS satellites. The precise GLONASS orbits stem from a combined processing of the IGS and IGLOS tracking network data.

\section{References}

Beutler G, Brockmann E, Gurtner W, Hugentobler U, Mervart L, Rothacher M (1994) Extended orbit modeling techniques at the CODE processing center of the International GPS Service for Geodynamics (IGS): theory and initial results. Manuscr Geod 19: $367-386$

Bock H, Hugentobler U, Beutler G (2003) Kinematic and dynamic determination of trajectories for low Earth satellites using GPS. In: Reigber $\mathrm{C}$ et al (eds) First CHAMP mission results for gravity, magnetic and atmospheric studies, Springer, pp 65-69

Boucher C, Altamimi Z, Sillard P (1999) The 1997 International Terrestrial Reference Frame (ITRF97). IERS tech note 27, Central Bureau of IERS, Observatoire de Paris, Paris

Brockmann E (1996) Combinations of solutions for geodetic and geodynamic applications of the global positioning system (GPS). PhD Dissertation, Astronomical Institute, University of Berne

Gooding RH (1971) Lumped fifteenth-order harmonics in the geopotential. Nature Phys Sci 231(25): 168

Habrich H (1999) Geodetic applications of the global navigation satellite system (GLONASS) and of GLONASS/GPS combinations. PhD Dissertation, Astronomical Institute, University of Berne

Hugentobler U (1998) Astrometry and satellite orbits: theoretical considerations and typical applications. Geodätisch-geophysikalische Arbeiten in der Schweiz, vol 57. Schweizerische Geodätische Kommission, Institut für Geodäsie und Photogrammetrie, Eidg. Technische Hochschule Zürich 
Hugentobler U, Ploner M, Schildknecht T, Beutler G (1999) Determination of resonant geopotential terms using optical observations of geostationary satellites. Adv Space Res 23(4): $767-770$

Hugentobler U, Schaer S, Fridez P (eds) (2001) Bernese GPS Software Version 4.2. Astronomical Institute, University of Berne

Hugentobler U, Schaer S, Springer T, Beutler G, Bock H, Dach R, Ineichen $\mathrm{D}$, Mervart $\mathrm{M}$, Rothacher $\mathrm{M}$, Wild $\mathrm{U}$, Wiget $\mathrm{A}$, Brockmann E, Weber G, Habrich H, Boucher C (2002) CODE IGS analysis center technical report. In: Gowey K (ed) IGS technical reports 2000. IGS Central Bureau, Jet Propulsion Laboratory, Pasadena, CA pp 73-82

Ineichen D, Rothacher M, Springer T, Beutler G (1999) Computation of precise GLONASS orbits for IGEX-98. In: Schwarz K-P (ed) Geodesy beyond 2000, the challenges of the first decade, IAG General Assembly, Birmingham, 19-30 July. International Association of Geodesy Symposia, vol 121. Springer, Berlin Heidelberg New York, pp 26-31

Ineichen D, Springer T, Beutler G (2001) Combined processing of the IGS and the IGEX network. J Geod 75: 575-586

Kaula WM (1966) Theory of satellite geodesy. Applications of satellites to geodesy. Blaisdell, Waltham, MA

Klokocnik J (1983) Orbital rates of Earth satellites at resonances to test the accuracy of Earth gravity field models. Celest Mech 30: 407-422

Rothacher M, Springer TA, Beutler G, Dach R, Hugentobler U, Ineichen D, Schaer S, Wild U, Wiget A, Brockmann E, Boucher C, Reinhard E, Habrich H (1999) Ann rep 1998 of the CODE analysis center of the IGS. In: Gowey K, Neilan R, Moore A (eds) IGS 1998 technical reports. IGS Central Bureau, Jet Propulsion Laboratory, Pasadena, CA, pp 61-73
Slater JA, Willis P, Beutler G, Gurtner W, Lewandowski W, Noll C, Weber R, Neilan RE, Hein G (1999) The international GLONASS experiment (IGEX-98): organization, preliminary results and future plans. In: Proc ION GPS-99, Nashville, TN, 14-17 September CD-ROM. The Institute of Navigation, 1800 Diagonal Foad, Suite 480, Alexandria, VA 22314, USA, pp 2293-2302

Schaer S (1999) Mapping and predicting the Earth's ionosphere using the Global Positioning System. Geodätisch-geophysikalische Arbeiten in der Schweiz, vol 59. Schweizerische Geodätische Kommission, Institut für Geodäsie und Photogrammetrie, Eidg. Technische Hochschule Zürich

Springer TA (2000) Modeling and validating orbits and clocks using the Global Positioning System. Geodätisch-geophysikalische Arbeiten in der Schweiz, vol 60. Schweizerische Geodätische Kommission, Institut für Geodäsie und Photogrammetrie, Eidg. Technische Hochschule Zürich

Springer TA, Beutler G, Rothacher M (1999) Improving the orbit estimates of the GPS satellites. J Geod 73(3): 147-157

Tapley BD, Watkins MM, Ries JC, Davis GW, Eanes RJ, Poole SR, Rim MJ, Schutz BE, Shum CK, Nerem RS, Lerch FJ, Marshall JA, Klosko SM, Pavlis NK, Williamson RG (1996) The joint gravity model 3. J Geophys Res 101(B12): 28 029-28 049

Willis P, Slater J, Beutler G, Gurtner W, Noll C, Weber R, Neilan RE, Hein G (1999) The IGEX-98 campaign: highlights and perspective. In: Schwarz K-P (ed) Geodesy beyond 2000, The challenges of the first decade. IAG General Assembly, Birmingham, 19-30 July. International Association of Geodesy Symposia, vol 121. Springer, Berlin Heidelberg New York, pp $22-25$ 